OPEN ACCESS

Edited by:

Marco Leonti,

Università degli Studi di Cagliari, Italy

Reviewed by:

Simona Pichini,

Istituto Superiore di Sanità, Italy

Francesco Paolo Busardò,

Sapienza Università di Roma, Italy

${ }^{*}$ Correspondence: Jacob M. Vigil

vigilj@unm.edu

Specialty section

This article was submitted to

Ethnopharmacology,

a section of the journal

Frontiers in Pharmacology

Received: 11 April 2018

Accepted: 26 July 2018

Published: 28 August 2018

Citation:

Stith SS, Vigil JM, Brockelman F,

Keeling $K$ and Hall B (2018)

Patient-Reported Symptom Relief

Following Medical Cannabis

Consumption

Front. Pharmacol. 9:916.

doi: 10.3389/fphar.2018.00916

\section{Patient-Reported Symptom Relief Following Medical Cannabis Consumption}

\author{
Sarah S. Stith ${ }^{1}$, Jacob M. Vigil2*, Franco Brockelman ${ }^{3}$, Keenan Keeling ${ }^{3}$ and \\ Branden Hall ${ }^{3}$ \\ 1 Department of Economics, The University of New Mexico, Albuquerque, NM, United States, ${ }^{2}$ Department of Psychology, \\ The University of New Mexico, Albuquerque, NM, United States, ${ }^{3}$ The MoreBetter Ltd., Washington, DC, United States
}

Background: The Releaf App ${ }^{\mathrm{TM}}$ mobile software application (app) data was used to measure self-reported effectiveness and side effects of medical cannabis used under naturalistic conditions.

Methods: Between 5/03/2016 and 12/16/2017, 2,830 Releaf App ${ }^{\mathrm{TM}}$ users completed 13,638 individual sessions self-administering medical cannabis and indicated their primary health symptom severity rating on an 11-point (0-10) visual analog scale in real-time prior to and following cannabis consumption, along with experienced side effects.

Results: Releaf $\mathrm{App}^{\mathrm{TM}}$ responders used cannabis to treat myriad health symptoms, the most frequent relating to pain, anxiety, and depressive conditions. Significant symptom severity reductions were reported for all the symptom categories, with mean reductions between 2.8 and 4.6 points (ds ranged from 1.29-2.39, ps < 0.001). On average, higher pre-dosing symptom levels were associated with greater reported symptom relief, and users treating anxiety or depression-related symptoms reported significantly more relief (ps < 0.001) than users with pain symptoms. Of the 42 possible side effects, users were more likely to indicate and showed a stronger correlation between symptom relief and experiences of positive (94\% of sessions) or a context-specific side effects (76\%), whereas negative side effects $(60 \%)$ were associated with lessened, yet still significant symptom relief and were more common among patients treating a depressive symptom relative to patients treating anxiety and pain-related conditions.

Conclusion: Patient-managed cannabis use is associated with clinically significant improvements in self-reported symptom relief for treating a wide range of health conditions, along with frequent positive and negative side effects.

Keywords: pain, anxiety, depression, cannabis, marijuana, quality of life, symptom management, side effects 


\section{INTRODUCTION}

Medicinal cannabis use is expanding rapidly in the United States, with thousands of new users daily, particularly older patients and people with significant health concerns, treating many different symptoms (Centers for Disease Control and Prevention, 2016; Han et al., 2016). Most patients have a wide variety of medicinal cannabis products available to them, ranging from traditional flower to edibles and tinctures. Naturalistic observational studies are generally well-suited for capturing how patients manage their treatment decisions in real-life, and how patient-managed cannabis therapies may contribute to symptom relief and potential side effects from use. Observational research designs allow patients to use the myriad Cannabis strains and cannabis-derived formulations (e.g., concentrates, tinctures, edibles, topicals, suppositories, toothpaste) made at home and/or commercially available and widely used in society, and can incorporate the breadth of health conditions for which medical cannabis has been sanctioned for use at the state-level. Lastly, observational studies also circumvent research barriers associated with cannabis' Schedule I status under United States federal law, which makes randomized controlled trials (RCTs) challenging to conduct (Stith and Vigil, 2016; National Academies of Sciences, Engineering, and Medicine, 2017).

Since its release in 2016, the commercially developed Releaf App $^{\mathrm{TM}}$ application (app; Releaf App, 2018) has been the only publically available, incentive-free patient educational software program designed for recording how individual cannabis usage sessions may correspond to immediate changes in primary symptom intensity levels and experienced side effects. This electronic assessment tool enables patients to monitor and manage their cannabis consumption decisions under naturalistic conditions while avoiding the limitations of retrospective survey collection methods (e.g., memory bias, social desirability effects). We used the Releaf $\mathrm{App}^{\mathrm{TM}}$ repository of over 2,830 patients and 13,368 individual cannabis administration sessions to examine two research questions: How does cannabis used under naturalistic conditions affect user-experienced symptom relief and side effects? Does the magnitude of experienced symptom relief and the prevalence of side effects vary across symptom categories? The results have clinical relevance for understanding how patient-managed medical cannabis therapies may correspond to changes in symptom intensity and potential side effects among people using cannabis for treating distinct health conditions (Hill and Weiss, 2016; Rubin, 2017).

\section{MATERIALS AND METHODS}

\section{Study Design}

A naturalistic observational research design, approved by the Institutional Review Board at the University of New Mexico, was used to analyze the Releaf $\mathrm{App}^{\mathrm{TM}}$ user-submitted data recorded between 5/03/2016 and 12/16/2017. Releaf $\mathrm{App}^{\mathrm{TM}}$ is a cross-platform (iOS and Android) mobile and tablet app backed by a secure cloud programming interface for capturing, processing, and storing anonymized user data. Out of 4,369 total users and 23,373 user interactions, we included only cannabis consumption sessions with reported starting symptom levels greater than 0 (on a $0-10,11$-point scale) and ending symptom levels reported within 90 min of the start of the session, resulting in a final sample of 2,830 users and 13,638 individual sessions for analysis. The Releaf $\mathrm{App}^{\mathrm{TM}}$ measures 27 possible negative symptom categories and 42 possible side effects. Symptoms were ultimately derived from qualifying conditions across medical cannabis programs in the United States, along with a few suggested by dispensaries and patients. The side effects (called "feelings" within the app) were crowd-sourced among Releaf $\mathrm{App}^{\mathrm{TM}}$ developers, beta testers, dispensaries, and patients, and included 19 positive, 12 negative, and 11 context-specific side effects available for selection. Supplementary Tables S1, S2 in the Supplemental Appendix provide descriptive statistics for all symptoms and side effects.

User sessions consist of a series of electronic instructions for recording characteristics of the cannabis medication (e.g., strain, potency, formulation), pre-dosing symptom severity rating along an 11-point visual analog facial pain scale from 0 (no detectable symptom level) to10 (severe), the timing of cannabis consumption, a post-dosing symptom severity rating, and the option to indicate any of the 42 listed side effects at any time during the session. Among our primary sample of users, 2,332 users reported side effects during 10,535 sessions.

\section{Study Outcomes}

Our goal was to calculate changes in patient-perceived symptom severity, the prevalence of positive and negative side effects associated with cannabis consumption, and whether the reported-effects differs depending on the symptom for which users were seeking treatment. We measured changes in symptom relief by subtracting the ending symptom level from the beginning symptom (possible range from -10 to 10 ). (Supplementary Figure S1 in the Supplemental Appendix provides a frequency table for each level of symptom relief.) Side effects were recorded as $\{0,1\}$ variables for whether the user selected that side effect from the menu. We categorize the side effects as positive, negative, or context-specific and then convert these categories of side effects into $\{0,1\}$ outcomes, count outcomes and outcomes measuring the portion of total available side effects in that category a user selected.

\section{Statistical Analysis}

We use means comparisons and least squares regression models to estimate the absolute and relative symptom changes and side effect profiles resulting from the cannabis user sessions. We also created an adjusted symptom relief profile score, the mean change in symptom levels plus the absolute number of listed negative side effects, to provide a relative metric of cost-benefit tradeoffs associated with cannabis use. Due to the small user counts for some of the reported symptoms, the large number of possible symptoms, and to facilitate interpretation in our regression analysis, we aggregate the most commonly reported symptoms across three broad symptom categories that included: Anxiety Symptoms (agitation/irritability, anxiety, insomnia, stress, and muscle spasms), Pain Symptoms (ten pain categories), and 
Depression Symptoms (depression). The remaining types of symptoms are less frequently reported or not clearly categorized. We also report the full regression results for the three categories of side effects (positive, negative, and context-specific) and the sign for regressions of symptom relief on the full range of 42 side effects. Standard errors are clustered at the user level to control for heteroskedasticity and arbitrary correlation.

\section{RESULTS}

Figure 1 shows the starting and ending symptom severity levels, the change in levels, the Cohen's $d$ of the difference, and the adjusted symptom relief profile score for each of the 27 discrete symptom categories. For all symptoms, the null hypothesis that the starting symptom severity level is less than or equal to the ending symptom severity can be rejected at the $p<0.001$ level. Using the adjusted symptom relief measure (symptom relief plus negative side effects), all but users with convulsions, dizziness, excessive appetite, or tremors experienced a net improvement in their symptom severity levels. Even for these symptoms, the adjusted mean symptom relief score still indicates a net benefit from use and the lack of a statistically significant change likely relates more to the small number of observations rather than the lack of an effect, given that these symptoms together constituted less than $3 \%$ of users and less than $1 \%$ of our sample. For all other symptoms, the null hypothesis of an increase or no change in the adjusted symptom relief score can be rejected at the $p<0.001$ level.

Table 1 provides additional information on starting and ending symptom severity levels, mean symptom relief, and the prevalence of positive, negative, and context-specific side effects by the aggregated symptom categories (anxiety, pain, and depression symptoms). For completeness, we include a fifth column including the remaining discrete symptom categories which did not fall under the three aggregated symptom categories. Little variation exists in starting and ending symptom levels and the symptom relief experienced, with the average user reporting a symptom decrease of 3.7. With regards to side effects, those with depression have a higher probability of reporting negative or context-specific side effects. The most common positive side effects are "relaxed" (64\%), "peaceful" (54\%), and "comfy" (38\%), the most common negative side effects are "dry mouth" (23\%), "foggy" (22\%), and "forgetful" (13\%) and the most common context-specific side effects are "high" (32\%), "sleepy" (27\%), and "thirsty" (27\%).

Table 2 examines how symptom relief varies across the broader symptom categories, with the constant representing the mean adjusted symptom change for the omitted category, (patients with pain-related symptoms). The first two regressions shown in Table 2 indicate that people with anxiety and depression report greater relief from using cannabis than people with chronic pain, and users with higher starting symptom levels report greater symptom relief. (The effects of cannabis on anxiety and depression symptoms are not statistically different from each other, although they are both greater than the effect of cannabis on pain-related symptoms). Negative responses or increases in symptom severity do occur, but the intercept in combination with the starting symptom level predicts that increases in symptom severity levels predominantly occur among users with starting symptoms equal to one. The third column in Table 2 shows that cannabis is more effective for anxiety and depression symptoms than for pain-related symptoms among patients reporting higher

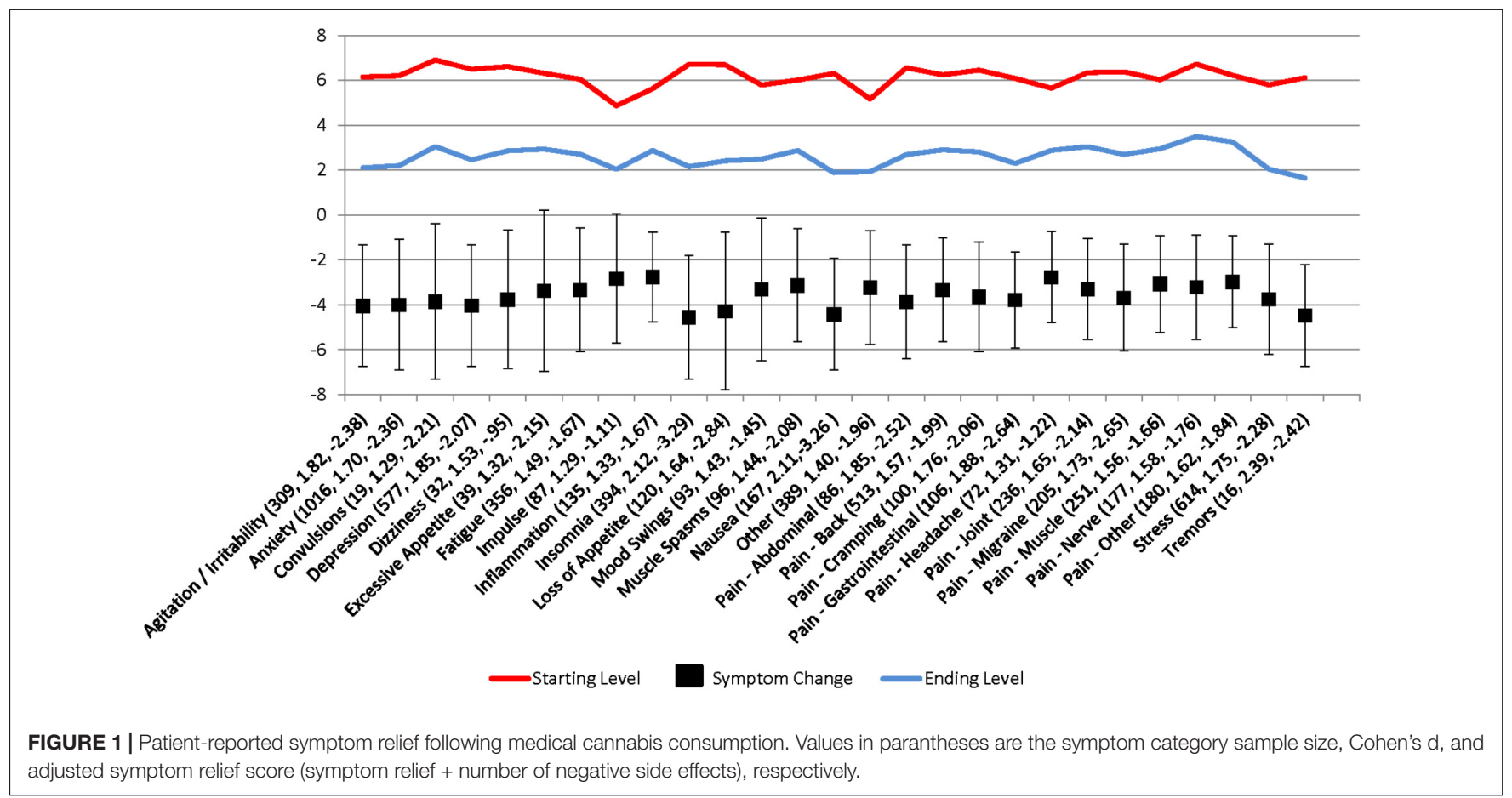


TABLE 1 | Descriptive statistics - symptom levels and experienced side effects.

\begin{tabular}{|c|c|c|c|c|c|}
\hline & Overall & Anxiety symptoms & Pain symptoms & Depression symptoms & Other \\
\hline N Sessions & 13638 & 5343 & 4267 & 1440 & 2588 \\
\hline N Users & 2830 & 1679 & 1223 & 577 & 1026 \\
\hline Starting symptom level & $6.2 \pm 2.2$ & $6.2 \pm 2.3$ & $6.3 \pm 2.0$ & $6.5 \pm 2.2$ & $5.8 \pm 2.4$ \\
\hline Ending symptom level & $2.5 \pm 2.2$ & $2.2 \pm 2.2$ & $3.0 \pm 2.1$ & $2.5 \pm 2.2$ & $2.4 \pm 2.3$ \\
\hline Symptom relief & $-3.7 \pm 2.6$ & $-4.0 \pm 2.8$ & $-3.3 \pm 2.3$ & $-4.0 \pm 2.7$ & $-3.4 \pm 2.8$ \\
\hline Better & $94.2 \%$ & $94.8 \%$ & $94.7 \%$ & $95.4 \%$ & $91.6 \%$ \\
\hline Same & $2.7 \%$ & $2.4 \%$ & $2.8 \%$ & $2.4 \%$ & $3.2 \%$ \\
\hline Worse & $3.1 \%$ & $2.8 \%$ & $2.5 \%$ & $2.2 \%$ & $5.2 \%$ \\
\hline Any positive side effect & $94.4 \%$ & $94.7 \%$ & $94.5 \%$ & $93.9 \%$ & $94.2 \%$ \\
\hline Any negative side effect & $60.0 \%$ & $60.0 \%$ & $58.9 \%$ & $65.5 \%$ & $58.8 \%$ \\
\hline Any context-specific side effect & $76.2 \%$ & $75.2 \%$ & $75.9 \%$ & $80.1 \%$ & $76.6 \%$ \\
\hline \# of positive side effects & $4.6 \pm 3.2$ & $4.6 \pm 3.2$ & $4.4 \pm 3.1$ & $4.8 \pm 3.4$ & $4.8 \pm 3.4$ \\
\hline \# of negative side effects & $1.4 \pm 1.7$ & $1.4 \pm 1.7$ & $1.3 \pm 1.6$ & $1.6 \pm 1.9$ & $1.3 \pm 1.7$ \\
\hline \# of context-specific side effects & $2.0 \pm 1.9$ & $2.0 \pm 1.9$ & $1.9 \pm 1.9$ & $2.1 \pm 1.9$ & $2.0 \pm 1.9$ \\
\hline$\%$ of positive side effects & $24 \%$ & $24 \%$ & $23 \%$ & $26 \%$ & $25 \%$ \\
\hline$\%$ of negative side effects & $11 \%$ & $11 \%$ & $10 \%$ & $13 \%$ & $10 \%$ \\
\hline$\%$ of context-specific side effects & $20 \%$ & $20 \%$ & $19 \%$ & $21 \%$ & $20 \%$ \\
\hline
\end{tabular}

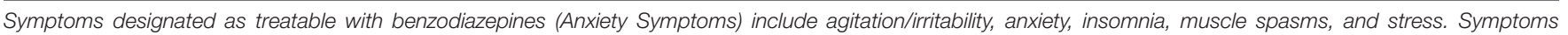
associated with Opioid treatment (Pain Symptoms) include all ten pain conditions. Depression is the only symptom designated as treatable with antidepressants.

TABLE 2 | Reported symptom relief for users treating anxiety, pain, and depression.

Outcome = symptom relief

\begin{tabular}{|c|c|c|c|}
\hline & (1) & (2) & (3) \\
\hline \multirow[t]{2}{*}{ Constant (opioid mean) } & $-3.309 * * *$ & $1.120 * * *$ & $0.355^{* *}$ \\
\hline & $(-3.459$ to -3.160$)$ & (0.804 to 1.436$)$ & (0.034 to 0.675$)$ \\
\hline \multirow[t]{2}{*}{ Anxiety symptoms } & $-0.704^{* * *}$ & $-0.763^{* * *}$ & $0.365^{*}$ \\
\hline & $(-0.944$ to -0.465$)$ & $(-0.953$ to -0.574$)$ & $(-0.062$ to 0.792$)$ \\
\hline \multirow[t]{2}{*}{ Depression symptoms } & $-0.723^{* * *}$ & $-0.563^{* * *}$ & $0.643^{*}$ \\
\hline & $(-1.060$ to -0.385$)$ & $(-0.817$ to -0.310$)$ & $(-0.021$ to 1.308$)$ \\
\hline \multirow[t]{2}{*}{ Starting symptom level $(1-10)$} & & $-0.706^{* * *}$ & $-0.582^{* * *}$ \\
\hline & & $(-0.757$ to -0.656$)$ & $(-0.639$ to -0.525$)$ \\
\hline \multirow[t]{2}{*}{ Anxiety*start } & & & $-0.181^{* * *}$ \\
\hline & & & $(-0.259$ to -0.102$)$ \\
\hline \multirow[t]{2}{*}{ Depression*start } & & & $-0.189^{* * *}$ \\
\hline & & & $(-0.305$ to -0.074$)$ \\
\hline Observations & 11,050 & 11,050 & 11,050 \\
\hline$R^{2}$ & 0.018 & 0.372 & 0.377 \\
\hline
\end{tabular}

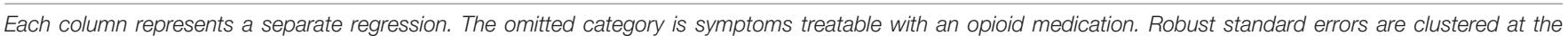
user level. The coefficients are reported in line with the variable names with confidence intervals below. Coefficients are reported with 95\% Confidence Intervals below. ${ }^{* * *} p<0.01,{ }^{* *} p<0.05,{ }^{*} p<0.10$.

symptom severity levels (A graphical representation of this relationship is presented in Supplementary Figure S2 in the Supplemental Appendix).

In order to take advantage of the full range of symptom categories available to Releaf $\mathrm{App}^{\mathrm{TM}}$ users, we also ran regressions including dummy variables for each of the symptoms, using back pain as the omitted category. After controlling for starting symptom level, clustering the standard errors at the user level, and using a statistical significance threshold of $p<0.05$, our results indicate that patients report greater symptom relief for treating agitation/irritability, anxiety, depression, excessive appetite, insomnia, loss of appetite, nausea, gastrointestinal pain, stress, and tremors than they do for treating back pain. Patients reported less symptom relief for treating impulsivity, headache, and nerve pain as compared to relief for treating back pain. The symptom relief for the other discrete symptom categories was indistinguishable from the reported symptom relief associated with back pain.

Table 3 explores whether patients using cannabis to treat pain, anxiety, or depressive symptoms differ in their experiences of positive, negative, or context-specific side effects. Chows tests (Chow, 1960) showed that users with anxiety-related symptoms 
TABLE 3 | Differences in side effect profiles across symptom categories.

\begin{tabular}{|c|c|c|c|}
\hline \multicolumn{4}{|c|}{ Outcome = side effect type } \\
\hline & Positive & Negative & Context-specific \\
\hline & \multicolumn{3}{|c|}{ Any } \\
\hline \multirow[t]{2}{*}{ Constant (opioid mean) } & $0.966^{* * *}$ & $0.496^{* * *}$ & $0.695^{* * *}$ \\
\hline & (0.942 to 0.989) & (0.428 to 0.565$)$ & (0.637 to 0.753 ) \\
\hline \multirow[t]{2}{*}{ Anxiety symptoms } & 0.001 & 0.013 & -0.006 \\
\hline & $(-0.012$ to 0.015$)$ & $(-0.033$ to 0.059$)$ & $(-0.049$ to 0.037$)$ \\
\hline \multirow[t]{2}{*}{ Depression symptoms } & -0.006 & $0.066^{* *}$ & $0.042^{*}$ \\
\hline & $(-0.029$ to 0.017$)$ & $(0.002$ to 0.131$)$ & $(-0.005$ to 0.090$)$ \\
\hline \multirow[t]{3}{*}{ Starting symptom level } & $-0.003^{*}$ & $0.015^{* * *}$ & $0.010^{* *}$ \\
\hline & $(-0.007$ to 0.000$)$ & (0.007 to 0.024$)$ & (0.002 to 0.019$)$ \\
\hline & \multicolumn{3}{|c|}{ Number } \\
\hline \multirow[t]{2}{*}{ Constant (opioid mean) } & $4.583^{* * *}$ & $1.081^{* * *}$ & $1.652^{* * *}$ \\
\hline & (4.013 to 5.154$)$ & (0.768 to 1.395$)$ & (1.356 to 1.947$)$ \\
\hline \multirow[t]{2}{*}{ Anxiety symptoms } & 0.182 & 0.077 & 0.077 \\
\hline & $(-0.100$ to 0.465$)$ & $(-0.104$ to 0.257$)$ & $(-0.113$ to 0.268$)$ \\
\hline \multirow[t]{2}{*}{ Depression symptoms } & $0.476^{*}$ & $0.324^{* *}$ & 0.134 \\
\hline & $(-0.010$ to 0.962$)$ & (0.053 to 0.596$)$ & $(-0.187$ to 0.454$)$ \\
\hline \multirow[t]{3}{*}{ Starting symptom level } & -0.035 & $0.036 * *$ & $0.044^{* *}$ \\
\hline & $(-0.142$ to 0.072$)$ & (0.000 to 0.072$)$ & (0.003 to 0.085$)$ \\
\hline & \multicolumn{3}{|c|}{ Percent of possible } \\
\hline \multirow[t]{2}{*}{ Constant (opioid mean) } & $0.241^{* * *}$ & $0.083^{* * *}$ & $0.165^{* * *}$ \\
\hline & (0.211 to 0.271$)$ & (0.059 to 0.107$)$ & (0.136 to 0.195$)$ \\
\hline \multirow[t]{2}{*}{ Anxiety symptoms } & 0.01 & 0.006 & 0.008 \\
\hline & $(-0.005$ to 0.024$)$ & $(-0.008$ to 0.020$)$ & $(-0.011$ to 0.027$)$ \\
\hline \multirow[t]{2}{*}{ Depression symptoms } & $0.025^{*}$ & $0.025^{* *}$ & 0.013 \\
\hline & $(-0.001$ to 0.051$)$ & (0.004 to 0.046$)$ & $(-0.019$ to 0.045$)$ \\
\hline \multirow[t]{2}{*}{ Starting symptom level } & -0.002 & $0.003^{* *}$ & $0.004^{* *}$ \\
\hline & $(-0.007$ to 0.004$)$ & (0.000 to 0.006$)$ & (0.000 to 0.009$)$ \\
\hline
\end{tabular}

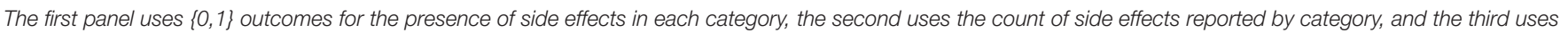

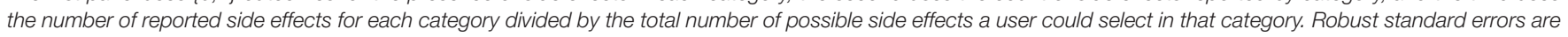
clustered at the user level. Coefficients are reported with 95\% Confidence Intervals below. ${ }^{* * *} p<0.01,{ }^{* *} p<0.05,{ }^{*} p<0.10$.

are no more or less likely than those with pain symptoms to report any of the three categories of side effects. Individuals with depression, however, are more likely to report negative and context-specific side effects than positive side effects. Higher starting symptom levels are also associated with more negative or context-specific side effect reporting and this relationship persists whether the side effect profile is defined as any of the side effects from that category of side effects, the number of side effects by category, or the percent of possible side effects in a category.

Table 4 tests whether different types of side effects are associated with differences in symptom relief. The results are robust across specifications; reporting positive or context-specific side effects is associated with greater symptom relief, while reporting negative side effects is associated with less symptom relief. For example, based on Column (4), a person with a starting symptom level of 5 who reports $100 \%$ of negative side effects would experience a 0.5 point increase in symptom severity on a 1-10 scale, whereas a similar user who does not report any negative side effects would experience 2.2 points of symptom relief, highlighting the importance of adjusting for starting symptom severity level and side effect profiles when evaluating the overall effectiveness of cannabis as a treatment modality.

\section{DISCUSSION}

This is the largest observational study to measure immediate changes in patient-reported symptom severity ratings and experienced side effects in real-time from using cannabis under naturalistic conditions. Building on previous research showing that cannabis may be an effective substitute for opioids (Hurd, 2016; Vigil et al., 2017) and other classes of prescription medications (e.g., sedatives; Piper et al., 2017; Stith et al., 2017), we provide evidence that cannabis is used to treat many different types of symptoms for which conventional pharmaceutical medications are typically prescribed, and that the magnitude of reported symptom relief and side effect profiles from using cannabis varies for people with different symptoms. 
TABLE 4 | Association of positive, negative, and context-specific side effects with symptom relief.

Outcome = symptom relief

(1)

(2)

(3)

(4)

\begin{tabular}{|c|c|c|c|c|}
\hline & $(-1.360$ to -0.841$)$ & $(-1.578$ to -1.111$)$ & (-3.046 to -1.643$)$ & $(-3.653$ to -2.145$)$ \\
\hline & (0.015 to 0.334$)$ & (0.192 to 0.480$)$ & (1.461 to 3.161$)$ & (2.045 to 3.498 ) \\
\hline Context-specific & $-0.339 * * *$ & $-0.239^{* * *}$ & $-0.781^{* *}$ & -0.417 \\
\hline Starting symptom level & & $(-0.710$ to -0.610$)$ & & $(-0.724$ to -0.608$)$ \\
\hline \multirow[t]{2}{*}{ Constant } & $-2.307^{* * *}$ & $1.894^{* * *}$ & $-3.098^{* * *}$ & $1.100^{* * *}$ \\
\hline & $(-2.625$ to -1.989$)$ & (1.441 to 2.348 ) & $(-3.372$ to -2.824$)$ & (0.818 to 1.382$)$ \\
\hline Observations & 10,535 & 10,535 & 10,535 & 10,535 \\
\hline$R^{2}$ & 0.015 & 0.349 & 0.036 & 0.376 \\
\hline
\end{tabular}

The first two columns measure use the existence of each category of side effect as independent variables, while the second two columns use the percent of possible in

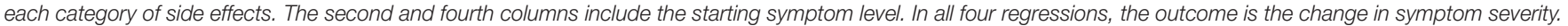
Robust standard errors are clustered at the user level. Coefficients are reported with $95 \%$ Confidence Intervals below. ${ }^{* * *} p<0.01,{ }^{* *} p<0.05,{ }^{*} p<0.10$.

The Releaf $\mathrm{App}^{\mathrm{TM}}$ users consumed cannabis to treat a wide range of health symptoms, the most frequent relating to pain, anxiety, or depression. Clinically and statistically significant reductions in patient-reported symptom severity levels existed in every single symptom category, suggesting that cannabis may be an effective substitute for several classes of medications with potentially dangerous and uncomfortable side effects and risky polypharmaceutical interactions, including opioids, benzodiazepines, and antidepressants (Weich et al., 2014; Centers for Disease Control and Prevention, 2016; Fontanella et al., 2016; Rudd et al., 2016; Sharma et al., 2016). Higher pre-dosing symptom levels were generally associated with greater post-dosing symptom relief and users treating an anxiety-related symptom or depression showed stronger symptom relief than users treating a pain symptom, even though depression is not a condition approved for medical cannabis use in most states.

Similar to clinical reviews showing that cannabis is associated with numerous, yet generally non-serious side effects (Wang et al., 2008; Whiting et al., 2016), positive and context-specific side effects were more commonly reported than negative side effects by the Releaf App ${ }^{\mathrm{TM}}$ users, with the most frequent reported side effects being positive (relaxed, peaceful, comfy) and the least frequent side effects being negative (paranoid, confused, headache). Positive side effect reporting was associated with the greatest reported symptom relief, followed by context-specific side effects, while negative side effects were associated with lower reported symptom relief. In general, patients treating depression were more likely to indicate a negative side effect than patients treating anxiety- or pain-related symptoms, though even users who reported only negative side effects reported significant decreases in moderate to severe symptom intensity levels after using cannabis.

One of the most striking patterns in the current results was the breadth of symptoms that appeared to improve following cannabis consumption. This pattern of responses could have been a function of characteristics of the software user interface (e.g., symptom intensity scale range), manner in which responders interacted with their mobile device (e.g., visual attention to common symptom severity levels), or with the systemic nature by which phytocannabinoids may affect the human mind and body. According to the endocannabinoid deficiency theory, many mental and physical health disturbances result from the dysregulation of the body's innate endocannabinoid system (ECS; Smith and Wagner, 2014; Di Marzo et al., 2015; Karhson et al., 2016; Russo, 2018), often described as a master network of chemical signals that promote somatic and psychological homeostasis, or psychobiological state-efficiency (BermudezSilva et al., 2010; Silvestri and Di Marzo, 2013; Acharya et al., 2017). The ECS consists of natural ligands (e.g., anandamide and 2-AG) and receptors (CB1 and $\mathrm{CB} 2$ ) that appear to play a major role in efficient regulation of a wide range of systems that include sleep, feeding (e.g., gut permeability and adipogenesis), libido and fertility, pain perception, motivation, happiness, anxiety, learning and memory, social functioning, autoimmune responses, cellular redox, and cancer pathophysiology (Valvassori et al., 2009; Muccioli et al., 2010; Abdel-Salam et al., 2012; Cani, 2012; Burstein, 2015; Du Plessis et al., 2015; McPartland et al., 2015; Karhson et al., 2016; Pava et al., 2016; Tegeder, 2016; Turcotte et al., 2016; Androvicova et al., 2017; Sierra et al., 2018). In other words, unlike conventional pharmaceutical approaches, which largely target specific neurotransmitter sites (e.g., monoamine neurotransmitter hypothesis; Delgado, 2000; Ng et al., 2015), cannabis may act to improve a broad spectrum of symptoms by regulating homeostatic functioning, perhaps best described as a system-modulating rather than symptom-modulating form of therapy.

Notwithstanding the strengths of the naturalistic research design and the potential implications of the study's findings, 
the study was limited primarily by the lack of a control group, e.g., non-cannabis users with the same symptom using a mobile device to indicate their immediate symptom intensity levels. There is also the potential confound of user-selection bias and exclusion of users that failed to complete sessions or even use the Releaf App ${ }^{\mathrm{TM}}$ due to a lack of symptom relief or negative side effects. (It is possible that selection bias could have worked in the opposite way, excluding patients that are already satisfied with their cannabis choices and therefore choose not to use the software app). This study chose to focus on the existence of symptom relief and side effects rather than offer clinical guidance as to which cannabis products offer preferential symptom relief and side effects profiles. As such we did not include product characteristics, e.g., routes of administration, quantity and method of ingestion, and cannabinoid content, all of which are likely crucial for understanding how cannabis affects symptom relief and side effect manifestation. We only show that, on average, most cannabis users experience symptom relief. Future research will benefit by incorporating these contextual factors into measurements of patient decisions and by dissecting how fundamental characteristics of the cannabis products themselves affect immediate and longer term changes in symptom relief and potential adverse consequences.

Patients with certain health conditions such as neurological disorders (e.g., multiple sclerosis, seizures, epilepsy, headache) may face differential risks for experiencing adverse effects or exacerbating their symptoms, for instance, depending on the amount of delta-9-tetrahydrocannabinol they consume, and caution should be used for patients considering using highly potent cannabis products (Solimini et al., 2017). Complicating matters are the allogamous (variable) and unstable nature of the Cannabis plant and the inherent inconsistencies in the chemical contents across plant batches and derived formulations, which are affected by genetic characteristics, but also environmental, cultivation, and storage conditions (Thomas and Pollard, 2016; Pacifici et al., 2017, 2018). These factors present challenges for both medical cannabis consumers and researchers as patients never have continuous access to cannabis products with precisely consistent chemotypes. Cannabis-based products (e.g., dried

\section{REFERENCES}

Abdel-Salam, O. M. E., El-Sayed El-Shamarka, M., Salem, N. A., ElDin, M., and Gaafar, A. (2012). Effects of Cannabis sativa extract on haloperidol-induced catalepsy and oxidative stress in the mice. EXCLI J. 11, 45-58.

Acharya, N., Penukonda, S., Shcheglova, T., Hagymasi, A. T., Basu, S., and Srivastava, P. K. (2017). Endocannabinoid system acts as a regulator of immune homeostasis in the gut. Proc. Natl. Acad. Ssci. U.S.A. 114, 5005-5010. doi: $10.1073 /$ pnas. 1612177114

Androvicova, R., Horace, J., Stark, T., Drago, F., and Micale, V. (2017). Endocannabinoid system in sexual motivational processes: is it a novel therapeutic horizon? Pharmacol. Res. 115, 200-208. doi: 10.1016/j.phrs.2016. 11.021

Bermudez-Silva, F. J., Viveros, M. P., McPartland, J. M., and Rodriguez de Fonseca, F. (2010). The endocannabinoid system, eating behavior and energy homeostasis: the end or a new beginning? Pharmacol. Biochemistry Behav. 95, 375-382. doi: 10.1016/j.pbb.2010.03.012 flower vs. oils) can differ in their dose reliability, and researchers have offered guidelines for dosing titration and experimental usage (Kahan et al., 2014; Pichini et al., 2018). However, until federal laws currently restricting pharmacodynamics research in the United States are reformed (Stith and Vigil, 2016) investigators still have tremendous opportunities to develop and incorporate innovative assessment tools, like the Releaf App ${ }^{\mathrm{TM}}$, into observational research designs for measuring how patients experience self-directed cannabis treatment in their normal everyday lives outside of clinical settings.

\section{AUTHOR CONTRIBUTIONS}

JV and SS conceived the study. FB, KK, and $\mathrm{BH}$ independently designed and developed the Releaf $A p^{T M}$ and server infrastructure as part of their effort to help create an education tool for medical cannabis patients. SS conducted the analyses. JV and SS drafted the manuscript. All authors contributed substantially to its intellectual content and revision.

\section{FUNDING}

This research was supported in part by the University of New Mexico Medical Cannabis Research Fund (mcrf.unm.edu).

\section{ACKNOWLEDGMENTS}

All authors had access to the data in the study and take responsibility for the integrity of the data and the accuracy of the data analyses.

\section{SUPPLEMENTARY MATERIAL}

The Supplementary Material for this article can be found online at: https://www.frontiersin.org/articles/10.3389/fphar. 2018.00916/full\#supplementary-material

Burstein, S. (2015). Cannabidiol (CBD) and its analogs: a review of their effects on inflammation. Bioorg. Med. Chem. 23, 1377-1385. doi: 10.1016/j.bmc.2015. 01.059

Cani, P. D. (2012). Crosstalk between the gut microbiota and the endocannabinoid system: impact on the gut barrier function and the adipose tissue. Clin. Microbiol. Infect. 18, 50-53. doi: 10.1111/j.1469-0691.2012.03866.x

Centers for Disease Control and Prevention (2016). Wide-Ranging Online Data for Epidemiologic Research (WONDER). Atlanta, GA: CDC.

Chow, G. C. (1960). Tests of equality between sets of coefficients in two linear regressions. Econometrica 28, 591-605. doi: 10.2307/1910133

Delgado, P. L. (2000). Depression: the case for a monoamine deficiency. J. Clin. Psychiatry 61(Suppl. 6), 7-11.

Di Marzo, V., Stella, N., and Zimmer, A. (2015). Endocannabinoid signaling and the deteriorating brain. Nat. Rev. Neurosci. 16, 30-42. doi: 10.1038/nrn 3876

Du Plessis, S. S., Agarwal, A., and Syriac, A. (2015). Marijuana, phytocannabinoids, the endocannabinoid system, and male fertility. J. Assist. Reprod. Genet. 32, 1575-1588. doi: 10.1007/s10815-015-0553-8 
Fontanella, C. A., Campo, J. V., Phillips, G. S., Hiance-Steelesmith, D. L., Sweeney, H. A., Tam, K., et al. (2016). Benzodiazepine use and risk of mortality among patients with schizophrenia: a retrospective longitudinal study. J. Clin. Psychiatry 77, 661-667. doi: 10.4088/JCP.15m 10271

Han, B. H., Sherman, S., Mauro, P. M., Martins, S. S., Rotenberg, J., and Palamar, J. J. (2016). Demographic trends among older cannabis users in the United States, 2006-13. Addiction 112, 516-525. doi: 10.1111/add.13670

Hill, K. P., and Weiss, R. D. (2016). Minimal physical health risk associated with long-term cannabis use-but buyer beware. JAMA 315, 2338-2339. doi: 10.1001/jama.2016.5181

Hurd, Y. L. (2016). Cannabidiol: swinging the marijuana pendulum from 'weed' to medication to treat the opioid epidemic. Trends Neurosci. 40, 124-127. doi: 10.1016/j.tins.2016.12.006

Kahan, M., Srivastava, A., Spithoff, S., and Bromley, L. (2014). Prescribing smoked cannabis for chronic noncancer pain: preliminary recommendations. Can. Fam. Physician 60, 1083-1090.

Karhson, D. S., Hardan, A. Y., and Parker, K. J. (2016). Endocannabinoid signaling in social functioning: an RDoC perspective. Transl. Psychiatry 6:e905. doi: 10. 1038/tp.2016.169

McPartland, J. M., Duncan, M., Di Marzo, V., and Pertwee, R. G. (2015). Are cannabidiol and $\Delta 9$-tetrahydrocannabivarin negative modulators of the endocannabinoid system? A systematic review. Br. J. Pharmacol. 172, 737-753. doi: 10.1111/bph.12944

Muccioli, G. G., Naslain, D., Bäckhed, F., Reigstad, C. S., Lambert, D. M., Delzenne, N. M., et al. (2010). The endocannabinoid system links gut microbiota to adipogenesis. Mol. Syst. Biol. 6:392. doi: 10.1038/msb.2010.46

National Academies of Sciences, Engineering, and Medicine (2017). Health and Medicine Division; Board on Population Health and Public Health Practice; Committee on the Health Effects of Marijuana: An Evidence Review and Research Agenda. Washington, DC: National Academies Press.

Ng, J., Papandreou, A., Heales, S., and Kurian, M. (2015). Monoamine neurotransmitter disorders - Clinical advances and future perspectives. Nat. Rev. Neurol. 11, 567-584. doi: 10.1038/nrneurol.2015.172

Pacifici, R., Marchei, E., Salvatore, F., Guandalini, L., Busardò, F. P., and Pichini, S. (2017). Evaluation of cannabinoids concentration and stability in standardized preparations of cannabis tea and cannabis oil by ultra-high performance liquid chromatography tandem mass spectrometry. Clin. Chem. Lab. Med. 55, 1555-1563. doi: 10.1515/cclm-2016-1060

Pacifici, R., Marchei, E., Salvatore, F., Guandalini, L., Busardò, F. P., and Pichini, S. (2018). Evaluation of long-term stability of cannabinoids in standardized preparations of cannabis flowering tops and cannabis oil by ultra-highperformance liquid chromatography tandem mass spectrometry. Clin. Chem. Lab. Med. 28, 94-96. doi: 10.1515/cclm-2017-0758

Pava, M. J., Makriyannis, A., and Lovinger, D. M. (2016). Endocannabinoid signaling regulates sleep stability. PLoS One 11:e0152473. doi: 10.1371/journal. pone. 0152473

Pichini, S., Pacifici, R., Busardò, F. P., Tagliabracci, A., and Giorgetti, R. (2018). The challenge of clinical application of FM2 cannabis oil produced in Italy for the treatment of neuropathic pain. Eur. Rev. Med. Pharmacol. Sci. 22, 863-865. doi: 10.26355/eurrev_201802_14363

Piper, B. J., DeKeuster, R. M., Beals, M. L., Cobb, C. M., Burchman, C. A., Perkinson, L., et al. (2017). Substitution of medical cannabis for pharmaceutical agents for pain, anxiety, and sleep. J. Psychopharmacol. 31, 569-575. doi: 10. $1177 / 0269881117699616$

Releaf App (2018). Available at: https://releafapp.com/ [accessed April 18, 2018].

Rubin, R. (2017). Medical marijuana is legal in most states, but physicians have little evidence to guide them. JAMA 317, 1611-1613. doi: 10.1001/jama.2017.0813

Rudd, R. A., Seth, P., David, F., and Scholl, L. (2016). Increases in drug and opioidinvolved overdose deaths-United States, 2010-2015. MMWR Morb. Mortal. Wkly. Rep. 65, 1445-1452. doi: 10.15585/mmwr.mm655051el

Russo, E. (2018). Clinical Endocannabinoid Deficiency (CECD): can this concept explain therapeutic benefits of cannabis in migraine, fibromyalgia, irritable bowel syndrome and other treatment-resistant conditions? Neuro Endocrinol. Lett. 29, 192-200.

Sharma, T., Guski, L. S., Freund, N., and Gøtzsche, P. C. (2016). Suicidality and aggression during antidepressant treatment: systematic review and metaanalyses based on clinical study reports. BMJ 352:i65. doi: 10.1136/bmj.i65

Sierra, S., Luquin, N., and Navarro-Otano, J. (2018). The endocannabinoid system in cardiovascular function: novel insights and clinical implications. Clin. Auton. Res. 1, 35-52. doi: 10.1007/s10286-017-0488-5

Silvestri, C., and Di Marzo, V. (2013). The endocannabinoid system in energy homeostasis and the etiopathology of metabolic disorders. Cell Metab. 17, 475-490. doi: 10.1016/j.cmet.2013.03.001

Smith, S. C., and Wagner, M. S. (2014). Clinical endocannabinoid deficiency (CECD) revisited: can this concept explain the therapeutic benefits of cannabis in migraine, fibromyalgia, irritable bowel syndrome and other treatmentresistant conditions? Neuro Endocrinol. Lett. 35, 198-201.

Solimini, R., Rotolo, M. C., Pichini, S., and Pacifici, R. (2017). Neurological disorders in medical use of cannabis: an update. CNS Neurol. Disord. Drug Targets 16, 527-533. doi: 10.2174/1871527316666170413105421

Stith, S. S., Vigil, J. M., Adams, I. M., and Reeve, A. P. (2017). Effects of legal access to cannabis on Scheduled II-V Drug Prescriptions. J. Am. Med. Dir. Assoc. 19, 59-64. doi: 10.1016/j.jamda.2017.07.017

Stith, S. S., and Vigil, J. M. V. (2016). Federal barriers to Cannabis research. Science 352:1182. doi: 10.1126/science.aaf7450

Tegeder, I. (2016). Endocannabinoids as guardians of metastasis. Int. J. Mol. Sci. 17:230. doi: 10.3390/ijms17020230

Thomas, B. F., and Pollard, G. T. (2016). Preparation and distribution of cannabis and cannabis-derived dosage formulations for investigational and therapeutic use in the United States. Front. Pharmacol. 7:285. doi: 10.3389/fphar.2016.00285

Turcotte, C., Blanchet, M., Laviolette, M., and Flamand, N. (2016). The CB2 receptor and its role as a regulator of inflammation. Cell. Mol. Life Sci. 73, 4449-4470. doi: 10.1007/s00018-016-2300-4

Valvassori, S. S., Elias, G., de Souza, B., Petronilho, F., Dal-Pizzol, F., Kapczinski, F., et al. (2009). Effects of cannabidiol on amphetamine-induced oxidative stress generation in an animal model of mania. J. Psychopharmacol. 25, 274-280. doi: 10.1177/0269881109106925

Vigil, J. M., Stith, S. S., Adams, I. M., and Reeve, A. P. (2017). Associations between medical cannabis and prescription opioid use in chronic pain patients: a preliminary cohort study. PLoS One 12:e0187795. doi: 10.1371/journal.pone. 0187795

Wang, T., Collet, J. P., Shapiro, S., and Ware, M. A. (2008). Adverse effects of medical cannabinoids: a systematic review. Can. Med. Assoc. J. 178, 1669-1678. doi: 10.1503/cmaj.071178

Weich, S., Pearce, H. L., Croft, P., Singh, S., Crome, I., Bashford, J., et al. (2014). Effect of anxiolytic and hypnotic drug prescriptions on mortality hazards: retrospective cohort study. BMJ 348:g1996. doi: 10.1136/bmj.g1996

Whiting, P. F., Wolff, R. F., Deshpande, S., Di Nisio, M., Duffy, S., Hernandez, A. V., et al. (2016). Cannabinoids for medical use: a systematic review and meta-analysis. JAMA 3913, 2456-2473.

Conflict of Interest Statement: The authors FB, KK, and BH were employed by company MoreBetter Ltd.

The remaining authors declare that the research was conducted in the absence of any commercial or financial relationships that could be construed as a potential conflict of interest.

Copyright (c) 2018 Stith, Vigil, Brockelman, Keeling and Hall. This is an open-access article distributed under the terms of the Creative Commons Attribution License (CC BY). The use, distribution or reproduction in other forums is permitted, provided the original author(s) and the copyright owner(s) are credited and that the original publication in this journal is cited, in accordance with accepted academic practice. No use, distribution or reproduction is permitted which does not comply with these terms. 